\title{
Rheological flow from a die and painting on a moving solid wall
}

\section{TANG Zemei \& HU Wenrui}

National Microgravity Laboratory/CAS, Institute of Mechanics, Chinese Academy of Sciences, Beijing 100080, China

Correspondence should be addressed to Tang Zemei (email: tang@nml.imech.ac.cn)

Received September 1, 2003

\begin{abstract}
Die swell is an important phenomenon in polymer processing, and is explained usually by rheological properties of the fluid. Because of the nonuniform of temperature distribution on the free surface of the liquid jet, the thermocapillary convection driven by surface tension gradient exists. The rheological fluid flowing out of a die and painting on a moving solid wall is studied by the numerical finite element method of a two-dimensional and unsteady model in the present paper, and both the rheological effect of a non-Newtonian fluid and the thermocapillary effect are considered. The results show that both effects will enlarge the cross-section of the fluid jet, and the rheological effect of non-Newtonian fluid dominates the process in general.
\end{abstract}

Keywords: die swell, thermocapillary flow, non-Newton flow, film jet.

DOI: $10.1360 / 03 y w 0034$

\section{Introduction}

The manufactures of fluid film and polymer require an understanding of the hydrodynamic process ${ }^{[1,2]}$. The enlarged cross-section of the fluid jet was observed in the polymer processing, where the die swell is an important phenomenon. The Swell or Die Swell effect is explained usually by rheological properties of the liquid medium. Tanner suggested a theory of Die-Swell, and assumed that the velocity vector has only one component along the jet ${ }^{[3]}$. Because of the non-uniformity of the cross-section, the flow field in the Die-Swell theory should be at least two-dimensional, as discussed in refs. [4, 5] for example. It is easy to believe that the difference of normal stresses can enlarge the cross-section of the melt jet, which, in addition, can also be contributed by the thermocapillary convection ${ }^{[6-8]}$. The experiments show that the solutal capillary flow induced by the surfactant of the liquid increases the thickness of the liquid layer ${ }^{[9]}$.

In the present paper, the enlargement of cross-section due to both the thermocapillary flow and rheological fluid is discussed for a jet liquid film model of a non-Newtonian fluid casting on a moving solid boundary. Both the rheological effect 
and thermocapillary effect can increase the cross-section of the liquid jet.

The heat transfer from melt region of high temperature to outside environment with lower temperature results in the temperature gradient on the free surface of the polymer jet. Therefore, the thermocapillary flow is induced, and will enlarge the cross-section of the film in the polymer processing. The thermocapillary effects on die swell in cases of Newtonian fluid and weak non-Newtonian fluid were investigated analytically in the previous papers ${ }^{[6,7]}$. These analyses are based on the approximations of lubrication theory and perturbation theory, and the high-order equations are used to solve analytically the heat and flow processes. When a liquid (or melt) film with relatively higher temperature is ejected from a vessel and painted on a moving solid film, the process is determined by the thermal hydrodynamic equations. The analytical solutions can be obtained in simplified conditions. The problem of Non-Newtonian fluid is usually complex, and it can only be solved by the numerical method.

The liquid film jetted from a die and painted on a moving solid wall is studied numerically in the present paper. The enlargement of cross-section, the flow field and temperature distribution of the liquid film are calculated in case of a two-dimensional and unsteady model. Both the rheological effect and thermocapillary flow are considered in the control equations and the boundary conditions. The physical and mathematical models of the non-Newtonian liquid film jet are described in the next section. In the third section, the calculation method is discussed. The computational results and discussions are included in the last section.

\section{Physical and mathematical models of non-Newtonian liquid film jet}

A simplified model is proposed as shown in fig. 1, where the liquid film of a melt or liquid is ejected from a nozzle vessel and then attached on a moving solid film of constant thickness. The Cartesian coordinate system $(x, y, z)$ is adopted, and a two-dimensional process $(x, z)$ is assumed with $\partial / \partial y=0$. The melt or liquid flows from the exit of the vessel to a long distance $l$. The initial height of liquid layer at $x=0$ is $h_{0}$. The liquid temperature $T_{0}$ at $x=0$ is higher than the environmental gas temperature $T_{\mathrm{g}}$

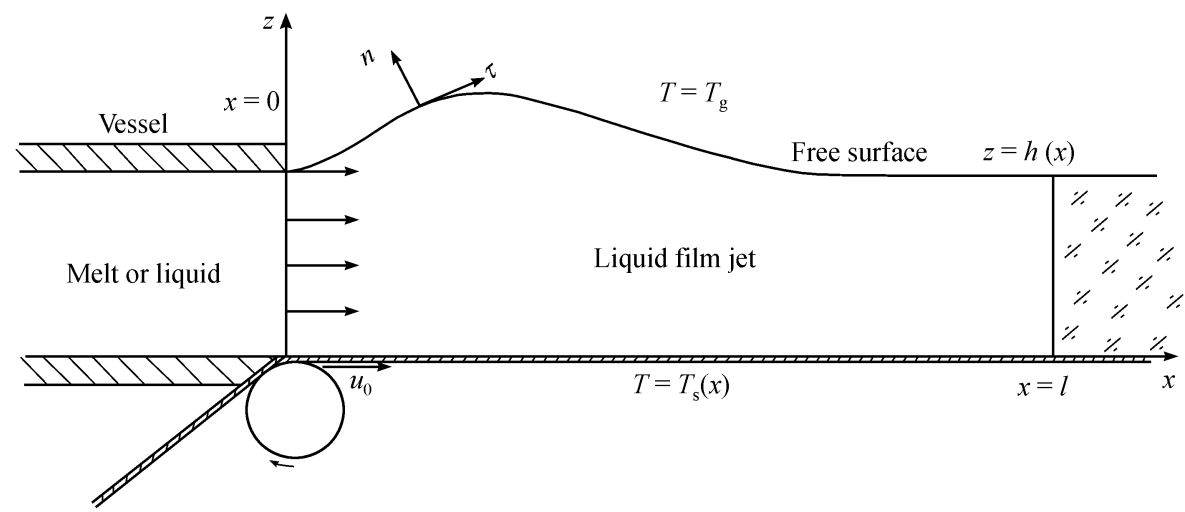

Fig. 1. Schematic diagram of the liquid jet ejected from a vessel and painted on a moving solid boundary. 
and the temperature is $T_{l}$ at $x=l$. A moving liquid film is touched smoothly with the lower boundary of the vessel, and moves with the velocity $u_{\mathrm{s}}$.

The liquid is considered as an incompressible fluid with constant viscosity and thermal diffusivity. The unsteady and two-dimensional conservation relationships are written as

$$
\begin{gathered}
\nabla \cdot v=0 \\
\frac{\partial v}{\partial t}+\rho v \cdot \nabla v=-\nabla p+\mu \nabla^{2} v+\nabla \pi \\
\frac{\partial T}{\partial t}+v \cdot \nabla T=\kappa \nabla^{2} T
\end{gathered}
$$

where $\rho, p, \mu, \kappa$ and $T$ are respectively the density, pressure, dynamic viscosity, heat diffusivity and temperature of the liquid, $(u, 0, w)$ is velocity vector $v$, and $\pi_{x x}, \pi_{x z}, \pi_{z x}$ and $\pi_{z z}$ are the components of a stress tensor $\pi$, where

$$
\begin{aligned}
& \pi_{x x}= 4 \alpha_{1}^{*}\left[\left(\frac{\partial u}{\partial x}\right)^{2}+\frac{1}{4}\left(\frac{\partial u}{\partial z}+\frac{\partial w}{\partial x}\right)^{2}\right] \\
&+2 \alpha_{2}^{*}\left[u \frac{\partial^{2} u}{\partial x^{2}}+w \frac{\partial^{2} u}{\partial x \partial z}+2\left(\frac{\partial u}{\partial x}\right)^{2}+\frac{\partial w}{\partial x}\left(\frac{\partial u}{\partial z}+\frac{\partial w}{\partial x}\right)\right], \\
& \pi_{x z}=2 \alpha_{2}^{*}\left[u \frac{\partial}{\partial x}\left(\frac{\partial u}{\partial z}+\frac{\partial w}{\partial x}\right)+w \frac{\partial}{\partial z}\left(\frac{\partial u}{\partial z}+\frac{\partial w}{\partial x}\right)+2\left(\frac{\partial u}{\partial x} \frac{\partial u}{\partial z}+\frac{\partial w}{\partial x} \frac{\partial w}{\partial z}\right)\right], \\
& \pi_{z z}=4 \alpha_{1}^{*}\left[\left(\frac{\partial w}{\partial z}\right)^{2}+\frac{1}{4}\left(\frac{\partial u}{\partial z}+\frac{\partial w}{\partial x}\right)^{2}\right] \\
&+2 \alpha_{2}^{*}\left[w \frac{\partial^{2} w}{\partial z^{2}}+u \frac{\partial^{2} w}{\partial x \partial z}+2\left(\frac{\partial w}{\partial z}\right)^{2}+\frac{\partial u}{\partial z}\left(\frac{\partial u}{\partial z}+\frac{\partial w}{\partial x}\right)\right],
\end{aligned}
$$

constants $\alpha_{1}^{*}$, and $\alpha_{2}^{*}$ are viscoelastic coefficients, and the conditions $\alpha_{1}^{*}>0, \alpha_{2}^{*}<0$ are satisfied. The first normal stress difference is $-2 \alpha_{2}^{*}$, and the second normal stress difference $\alpha_{1}^{*}+2 \alpha_{2}^{*}$ is small. Condition $\alpha_{1}^{*}+2 \alpha_{2}^{*}=0$ is assumed in the present paper.

The boundary conditions for the liquid layer can be written as follows:

$$
z=0: u=u_{\mathrm{s}}, w=0, T=T_{\mathrm{s}}(x)=T_{0}-\left(T_{0}-T_{l}\right)(x / l),
$$

where $T_{0}$ and $T_{l}$ are the temperatures respectively at the place $x=0$ and $x=l$. $z=h(x)$ at the free boundary of the fluid film: 


$$
\begin{gathered}
u \frac{d h}{d x}=w \\
p=\frac{2 \mu}{1+h^{\prime 2}(x)}\left[h^{\prime 2}(x) \frac{\partial u}{\partial x}-h^{\prime}(x)\left(\frac{\partial w}{\partial x}+\frac{\partial u}{\partial z}\right)+\frac{\partial w}{\partial z}\right] \\
+\frac{1}{1+h^{\prime 2}(x)}\left[h^{\prime 2}(x) \pi_{x x}-2 h^{\prime}(x) \pi_{x z}+\pi_{z z}\right]-\sigma \frac{h^{\prime \prime}(x)}{\left[1+h^{\prime 2}(x)\right]^{\frac{3}{2}}} \\
+\frac{-\sigma_{T}^{\prime} \mid}{\sqrt{1+h^{\prime 2}(x)}}\left(\frac{\partial T}{\partial x}+h^{\prime}(x) \frac{\partial T}{\partial z}\right) \\
+\frac{1}{\left(1+h^{\prime 2}(x)\right)}\left[2 h^{\prime}(x)\left(\frac{\partial w}{\partial z}-\frac{\partial u}{\partial x}\right)+\left(1-h^{\prime 2}(x)\right)\left(\frac{\partial u}{\partial z}+\frac{\partial w}{\partial x}\right)\right] \\
k \frac{\partial T}{\partial n}=-H\left(T-T_{\mathrm{g}}\right),
\end{gathered}
$$

where $h^{\prime}(x)=d h / d x, n$ is unit normal vector. $k$ and $H$ are respectively the heat conductivities of liquid and gas. The radiation effect is negligible in eq. (2.11), and the curvature of the free surface is $\frac{1}{R_{c}}=\frac{h^{\prime \prime}(x)}{\sqrt[3]{1+h^{\prime 2}(x)}}$.

The boundary conditions at $x=0$ are

$$
x=0: u=u_{0}\left[\frac{1}{4}-\left(\frac{z}{h_{0}}-\frac{1}{2}\right)^{2}\right], w=0 ; T=T_{0} .
$$

The flow is considered as parallel and laminar, and the velocity profile is in a parabolic distribution across the film. The original point $z=0$ is fixed at the moving solid film.

The boundary conditions at $x=l$ are

$$
x=l: \frac{\partial u}{\partial x}=0, \quad w=0, \quad T=0 .
$$

\section{Numerical method and the validation of the program}

Equations (2.1)-(2.3) can be rewritten as the non-dimensional expressions by using stream function and vorticity, which are solved under the initial condition and boundary conditions by the hybrid finite element method of fractional steps ${ }^{[10]}$. The 
characteristic line method is used for convection operator, and the diffusion item is calculated by the finite element method. The numbers of the cell are $121 \times 21$ in the directions $x, z$ respectively, and it means that the calculation domain is divided into 4800 triangular elements associated with 2541 nodes.

The steady solution of die swell problem is obtained by the unsteady calculation. The free surface at initial $t=0$ is adopted as a plane, and the initial velocity $u$ at $x=0$ is determined according to eq. (2.12), where $u_{0}=64.2 \mathrm{~mm} / \mathrm{s}$. The initial temperature is given as $T=0$ at $x=0$. After $20 \mathrm{~s}$ it is increased to $100^{\circ} \mathrm{C}$, and then keeps the constant temperature $T=T_{0}=100^{\circ} \mathrm{C}$ at $x=0$. In this case, the flow field and temperature distribution in the region of die swell can be calculated numerically under the given boundary conditions. Then, pressure distribution can be obtained from the velocity field, and the configuration of free surface is given by equilibrium condition of normal stress (2.9). The solutions of temperature and velocity coupled with the configuration of the free surface can be obtained at last.

The parameters are selected as follows in the present calculation:

$$
h_{0}=1 \mathrm{~mm}, \quad l=50 \mathrm{~mm}, \quad u_{0}=64.2 \mathrm{~mm} / \mathrm{s}, \quad u_{s}=0.3 u_{0}, \quad T_{0}=100^{\circ} \mathrm{C},
$$

and the related Reynolds number and Peclet number are

$$
\mathrm{Re}=0.642, \quad \mathrm{Pe}=2.03 \text {. }
$$

In order to check the validity of the program, the present program is used to calculate the thermocapillary convection in a square container. The calculated results are compared with those obtained by Bradley et al. The comparing results are shown in table 1 , where $-\psi_{\text {min }}$ is the minimum of stream function in the region, and $\omega_{\text {core }}$ is the vorticity at point $\left(x_{c}, z_{c}\right)$. The velocity at $x=0.5$ and $z=1$ is pointed by $u(0.5,1)$. Nusselt number is defined as $N u(x)=\int_{0}^{1}\left(M a \frac{\partial \psi}{\partial z} T-\frac{\partial T}{\partial x}\right) d z$.

Table 1 The comparison of Bradley's results ${ }^{[11]}(\mathrm{A})$ and the present results (B)

\begin{tabular}{cccccccccc}
\hline $\operatorname{Pr}=1$ & Grid & $\operatorname{Re}$ & $-\psi_{\text {min }}$ & $\omega_{\text {core }}$ & $x_{c}$ & $z_{c}$ & $u(0.5,1)$ & $N u_{x=0}$ & $N u_{x=1}$ \\
\hline A & $64 \times 64$ & $1.0 \times 10^{4}$ & $3.23 \times 10^{-3}$ & $-6.97 \times 10^{-2}$ & 0.58 & 0.62 & $2.96 \times 10^{-2}$ & 4.36 & 4.40 \\
B & $64 \times 64$ & $1.0 \times 10^{4}$ & $3.20 \times 10^{-3}$ & $-7.10 \times 10^{-2}$ & 0.58 & 0.63 & $2.95 \times 10^{-2}$ & 4.34 & 4.32 \\
\hline$P r=10$ & & & & & & & & & \\
\hline A & $64 \times 64$ & $1.0 \times 10^{3}$ & $2.60 \times 10^{-3}$ & $-10.0 \times 10^{-2}$ & - & - & $2.30 \times 10^{-2}$ & 3.79 & 3.83 \\
B & $64 \times 64$ & $1.0 \times 10^{3}$ & $2.75 \times 10^{-3}$ & $-9.56 \times 10^{-2}$ & - & - & $2.47 \times 10^{-2}$ & 3.97 & 3.89 \\
\hline
\end{tabular}

It is obvious that the present results given by the hybrid finite element method of fractional steps coincide with those given by Bradley, and the present calculation method and program are valid. 


\section{Results and discussions}

The configurations of the free surfaces with different non-dimensional rheological

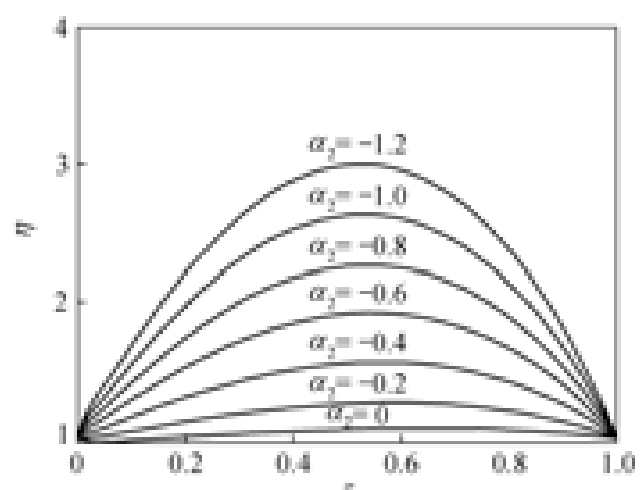

Fig. 2. Influence of rheological coefficient on the jet profiles, where the case $\alpha_{2}=0$ is related to the Newtonian fluid. coefficients $\alpha_{2}$ are shown in fig. 2, where $\eta=h(x) / H_{0}$ and $\xi=x / l$. Relationship $\alpha_{2}$ $=0$ means that only thermocapillary effect is included in the calculation. The rheological effect exits if $\alpha_{2}<0$. The smaller the value $\alpha_{2}$, the strong- er the rheological effect. It can be seen that both thermocapillary effect and rheolog- ical effect can enlarge the cross-section of the film jetting from vessel, and the crosssection can be enlarged several times due to the rheological effect. The non-dimensi- onal largest height of the cross-section depending on the non-dimensional rheological coefficient $-\alpha_{2}$ is shown in fig. 3 . The results show that the cross-section increases about $8 \%$ in case of Newtonian fluid $\left(\alpha_{2}=0\right)$ due to the thermocapillary effect, but it can be several times enlarged due to the rheological effect.

Fig. 4 shows the stream-lines (above) and temperature distributions (lower) for the case of thermocapillary effect only $\alpha_{2}$ $=0$ (left), and the case with both thermocapillary and rheological effects $\alpha$ $2=-0.8$ (right). The results show that a

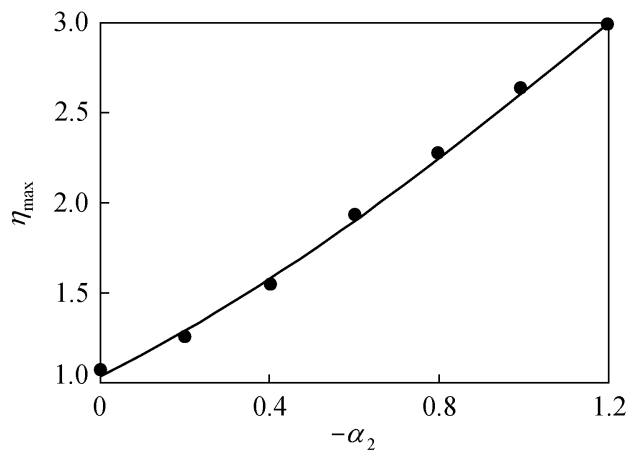

Fig. 3. Maximum cross-section of the liquid jet depending on the rheological coefficient $\alpha_{2}$. re-circle flow appears in the case of large stress difference if rheological effect is strong.

It is noted that the solutions depend on the expanding angle $d h(0) / d x$ at the exit of the vessel $x=0$. The expanding angle can only be determined by solving the problem coupling both the vessel region and the liquid film, and depends on the difference of normal stress in general. The larger expanding angle relates to the larger normal stress difference. The numerical calculations show that there is a critical expanding angle for a given coefficient $\alpha_{2}$, and the solution will be absent if the expanding angle is smaller than the critical one. The critical expanding angle $\eta^{\prime}(0)$ depending on the coefficient $\alpha_{2}$ is given in fig. 5, and the critical expanding angle is adopted in the calculation for a fixed rheological coefficient $\alpha_{2}$. In this case, fig. 5 shows the lowest effect of rheological fluid on the die swell. 


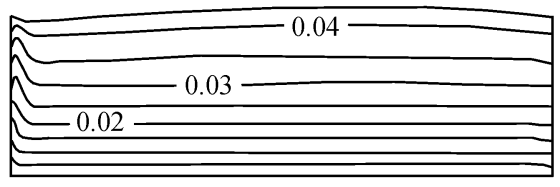

(a)

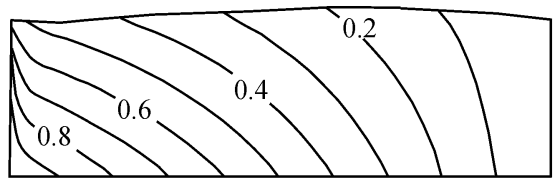

(c)

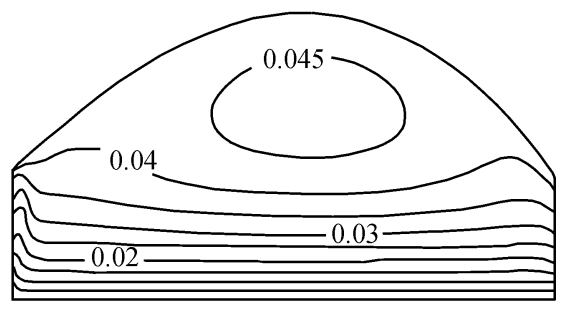

(b)

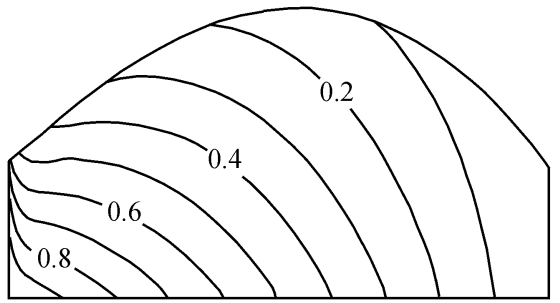

(d)

Fig. 4. The stream line (upper) and temperature distribution (lower) for the Newtonian fluid $\alpha_{2}=0$ (left) and the non-Newtonian fluid $\alpha_{2}=-0.8$ (right).

The problem about jet film of non-Newtonian fluid is solved numerically, where the temperature field is coupled with the hydrodynamic process of fluid. The results show that the thermocapillary effect due to heat transfer from the free boundary of the melt to the surrounding gas can enlarge the cross-section of the fluid jet. Furthermore, the pressure distribution depends mainly on the rheological property of the nonNewtonian fluid, which enlarges the

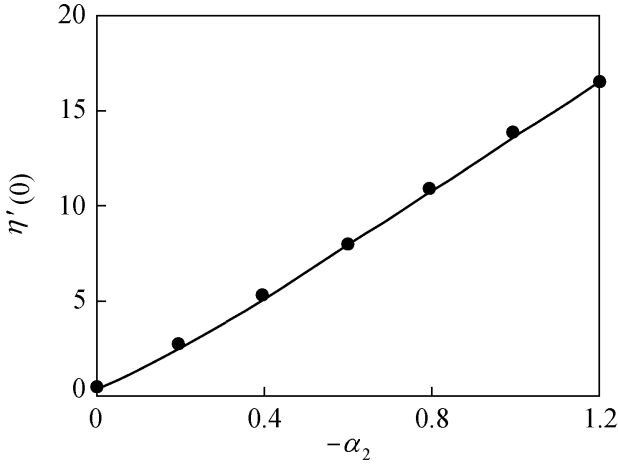

Fig. 5. Critical expanding angle depending on the rheological coefficient $\alpha_{2}$. cross-section of the fluid jet too. The reason of enlarging the crosses-section is considered as the coupled effects of the thermocapillary effect and rheological effect, and the rheological effect is more important than the thermocapillary one in general.

A non-Newtonian fluid of second-order fluid model is assumed in the present paper, and this model has obvious limitations. However, polymers are various, and the Newtonian fluid can even describe some sorts of the polymers. The discussion of the present paper will only be applied to the sorts of polymers, which can be described approximately by the second-order fluid model. The results of the present paper show that, similar to the case of Newtonian fluid, the heat transfer may induce the thermocapillary flow and may increase the cross-section of the fluid jet, although the effect of non-Newtonian fluid is more important. 
The Barus effect can have enlargement of cross-section such as two or three times larger than the cross-section of the vessel exit in real cases, and the results of the present paper give an explanation. More studies should be conducted, especially in connection with the manufacture processing, and more attention should be paid to study the rheological fluid of other models related to the real cases of polymer application.

\section{References}

1. Tucker, C. L., Computer Modeling for Polymer Processing, New York: Hanser, 1989, 370-372.

2. Silagy, D., Demay, Y., Agussant, J. F., Stationary and stability analysis of the film casting process, J. Non-Newtonian Fluid Mechanics, 1998, 79: 563-570.

3. Tanner, R. I., A theory of Die-Swell, J. Polymer Science: Part A-2, 1970, 3: 2067-2072.

4. Bohme, G., Die swell, in Non-Newtonian Fluid Mechanics (ed. Böhme, G.), Amsterdam: North-Holland, 1987, 4.3.

5. Agassant, J. F., Avenas, P., Sergant, J. Ph. et al., Extrudate swell, in Polymer Processing (eds. Agassant, J. F., Avenas, P., Sergant, J. Ph. et al.), New York: Hanser, 1991, 273-276.

6. Hu, W. R., Imaishi, N., Thermocapillary flow in a jet of liquid film painted on a moving boundary, Langmuir, 2000, 16: 4632-4638.

7. Hu, W. R., Imaishi, N., Thermocapillary flow in an annular liquid layer painted on a moving fiber, International J. Heat and Mass Transfer, 2000, 43: 4457-4466.

8. Hu, W. R., Hui, H. W., Rheological effect on thermocapillary flow of liquid film jet printed on a moving boundary, Science in China, Ser. A, 2002, 45(9): 1171-1182.

9. Ramdaul, O. O., Quere, D., Thickening factor in Marangoni coating, Langmuir, 1997, 13: 2911—2914.

10. Wu, J. H., A hybrid method of fractional steps with $L_{\infty}$-stability for numerical modeling of aquatic environments, Advances in Hydrodynamics, 1985, 1: 27-36.

11. Carpenter Bradley, M., Homsy, G. M., High Marangoni number convection in a square cavity: Part II, Phys. Fluids, Ser. A, 1990, 2(2): 137-149. 